\title{
ON THE COHOMOLOGY OF KÄHLER GROUPS
}

\author{
BRUNO KLINGLER
}

\section{Contents}

1. Introduction and results

2. Continuous cohomology of real simple Lie groups

3. Characteristic classes of representations

4. Non-Abelian Hodge theory

5. Geometry of period domains

6. First Pontryagin class : proof of theorem 4

7. Proof of corollary 1 and theorem 1

8. Proof of theorem 2 and theorem 3

References

\section{InTRODUCTION AND RESULTS}

1.1. The main result. This is the second of two papers (c.f. [20]) investigating which finitely generated groups can be realized as fundamental groups of a connected compact Kähler manifold. Such groups are called Kähler groups. Even if this question is completely open there are many known obstructions for an infinite finitely presented group being Kähler (we refer to [1] for a panorama). Most of them come from Hodge theory (Abelian or not) in cohomological degree one. The most interesting conjecture concerning infinite Kähler groups, due to Carlson and Toledo and publicized by Kollar 22] and Simpson, deals with cohomology in degree 2 :

Conjecture 1 (Carlson-Toledo). Let $\Gamma$ be an infinite Kähler group. Then virtually $b^{2}(\Gamma)>0$.

Remark 1. Recall that a group $\Gamma$ has virtually some property $\mathcal{P}$ if a finite index subgroup $\Gamma^{\prime} \subset \Gamma$ has $\mathcal{P}$.

Our first result in this paper is the following :

Theorem 1. Let $\Gamma$ be a finitely presented group admitting a morphism $\rho: \Gamma \longrightarrow G L(n, \mathbb{C})$ with unbounded image in $G L(n, \mathbb{C})$.

If $\Gamma$ is a Kähler group then $b^{2}(\Gamma)+b^{4}(\Gamma)>0$.

Moreover if $b^{2}(\Gamma)=0$ then the bounded cohomology group $H_{b}^{4}(\Gamma, \mathbb{R})$ is non-trivial.

Let us make some comments on theorem 1

- In 31] Toledo constructed infinite Kähler groups (in fact : fundamental groups of complex smooth projective varieties) which are not residually finite. In particular such groups are not linear. However all known infinite Kähler groups satisfy the assumption of theorem [1: they admit a morphism to some complex linear group $G L(n, \mathbb{C})$ with unbounded image. 
- Restricting ourselves to such groups rather than infinite groups is a classical drawback in the study of Kähler groups : we know nothing about their non-linear part.

- The main content of theorem 1 is of course that $b^{2}(\Gamma)+b^{4}(\Gamma)>0$. Although the remark concerning bounded cohomology might be of interest I don't know many groups $\Gamma$ for which one can compute $H_{b}^{4}(\Gamma, \mathbb{R})$.

1.2. A variant of [20, theor.1]. In [20] we proved the following :

Theorem. [20, theor.1] Let $\Gamma$ be an infinite linear $r$-dimensional duality group, $r \geq 6$. If $\Gamma$ is a Kähler group then virtually

$$
b^{2}(\Gamma)+b^{4}(\Gamma)>0 .
$$

Theorem 1 may look more general than [20, theor.1] : there is no duality nor linearity assumption, and one does not have to pass to a finite index subgroup. However it is crucial to realize that theorem 1 does not imply [20, theor.1] as there are many linear groups whose complex representations are all bounded. Typically [20, theor.1] implies the following :

Theorem. [20, theor.2] Let $G_{v}$ be the group of $F_{v}$-points of an algebraic group $\mathbf{G}_{v}$, with reductive neutral component, over a non-Archimedean local field $F_{v}$ of characteristic 0 . Suppose that $\operatorname{rank}_{F_{v}} \mathbf{G}_{v} \geq 6$.

Then a cocompact lattice $\Gamma \subset G_{v}$ is never the fundamental group of a smooth projective complex variety.

On the other hands theorem 1 says nothing in this situation : such a $\Gamma \subset G_{v}$ is linear but every representation $\rho: \Gamma \longrightarrow G L(n, \mathbb{C})$ has bounded image by Margulis's superrigidity theorem [23].

It is better to think of theorem [1, respectively [20, theor.1] as the geometric facet, respectively the topological facet, of the same phenomenon. As we will see the main ingredient for theorem 1 is non-Abelian Hodge theory and a careful differential-geometry study of Pontryagin forms on period domains. On the other hand [20, theor.1] relies on the topological properties of Stein spaces and duality properties in group cohomology.

In fact putting together theorem 1 and the proof of [20, theor.1] we obtain the following improvement of theorem 1 for linear duality groups :

Theorem 2. Let $\Gamma \subset G L(n, \mathbb{C})$ be an infinite linear $r$-dimensional duality group, $r \geq 6$, with $b^{2}(\Gamma)=0$. Suppose that $\Gamma$ is not bounded in $G L(n, \mathbb{C})$. If $\Gamma$ is Kähler then virutally $b^{4}(\Gamma)>1$.

1.3. Application to lattices in real Lie groups. A simple way of constructing "big" Kähler groups (and essentially the only known one) consists in generalizing to higher dimensions the uniformization of a smooth projective complex curve of genus $g \geq 2$ as quotient of the Poincaré disk. Let $G$ be a real simple Lie group of Hermitian non-compact type, i.e. such that the associated symmetric space $G / K$ admits an unbounded $G$-invariant Kähler metric. For example $G=P U(n, 1)$ the group of isometries of the unit ball $\mathbf{B}_{\mathbb{C}}^{n}=\left\{\left[z_{1}, \cdots z_{n+1}\right] \in \mathbf{P}^{n} \mathbb{C}, \quad\left|z_{1}\right|^{2}+\cdots+\left|z_{n}\right|^{2}<\left|z_{n+1}\right|^{2}\right\}$. Any torsion free cocompact lattice $\Gamma$ of $G$ acts properly discontinuously on $G / K$ and the quotient $X=\Gamma \backslash G / K$ is a compact Kähler manifold (in fact a smooth projective complex variety) with fundamental group $\Gamma$. One can construct more sophisticated examples starting from these ones using Lefschetz's hyperplane theorem and glueing techniques [31.

In this context theorem 2 enable us to prove (with some restrictions on the rank) another conjecture by Carlson-Toledo (certainly the main motivation for conjecture 1) : 
Theorem 3. Let $G$ be a simple real Lie group of non-compact type, of real rank at least 20, and $\Gamma$ a cocompact lattice in $G$. Then $\Gamma$ if a Kähler group if and only if $G$ is of Hermitian type.

Remark 2. In 27] Simpson showed that if a cocompact lattice $\Gamma$ in a simple real Lie group $G$ is Kähler then $G$ is necessarily of Hodge type (i.e. admits discrete series). Thus theorem 3 consists in reducing the class of groups of Hodge type to the class of groups of Hermitian type. That is : excluding the two families $G=S O(2 p, q), p \neq 1$ and $G=S p(p, q), p \geq q, q \geq 1$ and eight exceptional groups. Our proof does not apply in small rank. Certainly the most interesting remaining case is the following : does there exists a cocompact lattice in $S p(n, 1)$ which is a Kähler group ?

1.4. Ingredients for the proof. Let $\Gamma$ be the fundamental group of a compact connected Riemannian manifold $X$ and $\rho: \Gamma \longrightarrow G=\mathbf{G}(\mathbb{R})$ be a Zariski-dense representation, where $\mathbf{G}$ denotes a real almost simple algebraic group (non-anisotropic). One can try (c.f. [19]) to understand $\rho$ by studying the pull-back map in cohomology

$$
\rho^{*}: H_{c t}^{\bullet}(G, \mathbb{R}) \longrightarrow H^{\bullet}(\Gamma, \mathbb{R})
$$

The continuous cohomology $H_{c t}^{\bullet}(G, \mathbb{R})$ is completely understood since the $60^{\prime} s$. It is naturally isomorphic to the De Rham cohomology $H^{\bullet}\left(A^{\bullet}(G / K)^{G}, d\right)$ of $G$-invariant differential forms on the symmetric space $G / K$ or equivalently with the relative Lie algebra cohomology $H^{\bullet}(\mathfrak{g}, K, \mathbb{R})$. On the other hand the datum of $(X, \rho)$ essentially uniquely defines an harmonic $\rho$-equivariant map

$$
\overline{f_{\rho}}: \tilde{X} \longrightarrow G / K,
$$

where $\tilde{X}$ denotes the universal cover of $X$. Contemplating the commutative diagram

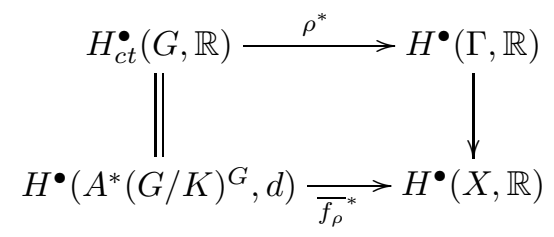

we would like to exhibit some class $c \in H_{c t}^{\bullet}(G, \mathbb{R})$ whose image ${\overline{f_{\rho}}}^{*}(c)$ does not vanish in $H^{\bullet}(X, \mathbb{R})$, thus showing that the class $\rho^{*}(c) \in H^{\bullet}(\Gamma, \mathbb{R})$ does not vanish. For a general $X$ not much can be done. When $X$ is a compact Kähler manifold this picture can be much improved using non-Abelian Hodge theory.

Let $X$ and $\rho$ be as in theorem1. If $\Gamma$ admits a non-rigid complex finite dimensional representation then Simpson's version of Lefschetz's theorem readily implies that $b^{2}(\Gamma)>0$ (c.f. [20, Appendix $\mathrm{B}]$ ). Thus we can assume that $\rho$ is reductive rigid. As $\rho$ is unbounded non-Abelian Hodge theory implies that $\rho$ is the monodromy of a (polarized) non-trivial complex variation of Hodge structure (CVHS for short) on $X$ : there exists a $C^{\infty}$ complex vector bundle $E$ (say of rank $n$ ) with a decomposition $E=\oplus_{i=0}^{k} E^{i}$ (called the Hodge decomposition) into a direct sum of $C^{\infty}$ subbundles, a flat connection $\nabla$ with monodromy $\rho$ satisfying Griffiths's transversality condition

$$
\nabla: E^{i} \longrightarrow A_{X}^{0,1}\left(E^{i+1}\right) \oplus A_{X}^{1,0}\left(E^{i}\right) \oplus A_{X}^{0,1}\left(E^{i}\right) \oplus A_{X}^{1,0}\left(E^{i-1}\right)
$$

and a parallel Hermitian form $h$ which makes the Hodge decomposition orthogonal and is positive on $E^{i}$ if $i$ is even and negative if $i$ is odd. If we set $r_{i}=\operatorname{rk} E^{i}$, the monodromy representation $\rho$ has image contained in a group isomorphic to $U(p, q) \subset G L(n=p+q, \mathbb{C})$ where $p=\sum_{i \text { even }} r_{i}$ and $q=\sum_{i \text { odd }} r_{i}$. 
The Hodge filtration of $E$ by the subbundles $F^{s}=\oplus_{i \geq s} E^{i}$ is holomorphic. Let us fix $x_{0} \in X$ and let $\mathbb{E}=\oplus_{i=0}^{k} \mathbb{E}^{i}$ be the fiber of $E$ over $x_{0}$ endowed with the restriction $h_{0}$ of the Hermitian metric $h$ on $E$. Let $\mathbf{G}_{\mathbb{C}}$ be the complex reductive algebraic group Zariski-closure of $\rho(\Gamma)$ in $\mathbf{P G L}(\mathbb{E})$, let $\mathbf{G}$ be its real form $\mathbf{G}_{\mathbb{C}} \cap \mathbf{P} \mathbf{U}\left(\mathbb{E}, h_{0}\right)$, let $Q$ be the parabolic subgroup of $G_{\mathbb{C}}=\mathbf{G}_{\mathbb{C}}(\mathbb{C})$ which stabilizes the flag $\mathbb{F}^{k} \subset \mathbb{F}^{k-1} \subset \cdots \subset \mathbb{F}^{1} \subset \mathbb{F}^{0}=\mathbb{E}$ defined by the Hodge filtration and let $V=G \cap Q \subset \mathrm{P}\left(U\left(\mathbb{E}^{0}, h_{0 \mid \mathbb{E}^{0}}\right) \times \cdots \times U\left(\mathbb{E}^{k}, h_{0 \mid \mathbb{E}^{k}}\right)\right)$. We obtain a period domain $D=G / V$ in the flag manifold $\check{D}=G_{\mathbb{C}} / Q$. Summarizing, considering the classifying map for the $\mathbb{C}$ HS $E$ on $X$ we obtain :

- a real form $\mathbf{G}$ of Hodge type of the Zariski-closure $\mathbf{G}_{\mathbb{C}}$ of $\rho(\Gamma)$ in $\mathbf{G L}(n, \mathbb{C})$ such that $\rho(\Gamma) \subset G=\mathbf{G}(\mathbb{R})$.

- a period domain $G / V$ for $G$ and a holomorphic $\rho$-equivariant period map $f_{\rho}: \tilde{X} \longrightarrow G / V$ making the following diagram commutative :

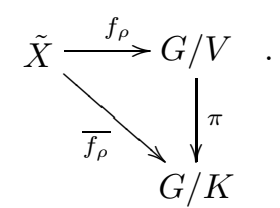

We can assume without loss of generality that the group $\mathbf{G}$ is $\mathbb{R}$-simple. For a simple real Lie group $G$ of Hodge type the continuous cohomology $H_{c t}^{\bullet}(G, \mathbb{R})$ concentrates in even degree. Cohomology in degree 2 is not useful for a general group of Hodge type : it is non-zero if and only if $G$ is of Hermitian type (in which case the $G$-invariant Kähler form $\omega_{G / K}$ generates $H_{c t}^{2}(G, \mathbb{R})$ ). On the other hand the cohomology $H_{c t}^{4}(G, \mathbb{R})$ is always non-trivial for $G$ of Hodge type : it contains the first Pontryagin form $p_{1}(\mathcal{F}) \in H^{4}(\mathfrak{g}, K, \mathbb{R})$ of the real oriented $\mathcal{C}^{\infty}$ vector bundle

$$
\mathcal{F}:=G \times_{K, \text { Ad }} \mathfrak{k}
$$

on $G / K$ associated to the $K$-principal bundle $K \hookrightarrow G \longrightarrow G / K$, the adjoint representation Ad $: K \longrightarrow \operatorname{Aut}(\mathfrak{k})$. Here the form $p_{1}(\mathcal{F})$ is computed with respect to the standard $G$-invariant Riemannian metric on $\mathcal{F}$ coming from the Killing form on $\mathfrak{k}$. The main idea for proving theorem 1 consists in studying the pull-back of this class under $\overline{f_{\rho}}$ using the factorization through the holomorphic horizontal map $f_{\rho}$. We prove the following :

Theorem 4. Let $\rho: \Gamma=\pi_{1}(X) \longrightarrow G$ be the monodromy of a complex variation of Hodge structure $f_{\rho}: \tilde{X} \longrightarrow G / V$ on a compact Kähler manifold $X$. Suppose that $\rho(\Gamma)$ is unbounded in $G$.

Then the $(2,2)$-form ${\overline{f_{\rho}}}^{*} p_{1}(\mathcal{F}) \in A^{2,2}(X, \mathbb{R})$ is non-positive on $X$.

In particular the class $\rho^{*} p_{1}(\mathcal{F}) \in H^{4}(\Gamma, \mathbb{R})$ is non-zero except if ${\overline{f_{\rho}}}^{*} p_{1}(\mathcal{F})$ vanishes identically on $X$.

Some comments on theorem 4 :

- As far as I know the only non-trivial case of theorem 4 which was previously deals with $G=$ $S p(n, 1)$. In this case $G / V$ is the twistor space of the quaternionic symmetric space $G / K$ and the quaternionic form $p_{1}(\mathcal{F})$ pulls back via $\pi$ to $-c_{1}(L)^{2}$, where $L$ is a holomorphic $G$-equivariant Hermitian line bundle on $G / V$. Moreover $L$ is seen to be positively curved in the horizontal directions [25]. Thus in this particular case the form $\pi^{*} p_{1}(\mathcal{F})$ is already non-positive on pairs of horizontal vectors.

- for the general case however theorem 4 uses the full-strength of the definition of a CVHS : the form $\pi^{*} p_{1}(\mathcal{F})$ is shown to be non-positive only on pairs of commuting vectors in the 
horizontal directions. Thus one crucially uses the classical fact that the image of $d f_{\rho}(x)$ for any point $x \in \tilde{X}$ can be canonically identified with an abelian horizontal subalgebra of $\mathfrak{g}_{\mathbb{C}}$.

To deduce theorem 1 from theorem 4 it is enough to understand what happens in the case where the $(2,2)$-form ${\overline{f_{\rho}}}^{*} p_{1}(\mathcal{F}) \in A^{2,2}(X, \mathbb{R})$ vanishes identically on $X$. This case looks very peculiar. It happens of course if the map $f_{\rho}$ factorizes through a curve but it seems there are other cases which deserve further study. However for our purpose it is enough to argue as follows. Given $E=\oplus_{i=0}^{k} E^{i}$ our $\mathbb{C V H S}$ on $X$ let $Q_{k} \supset Q$ be the parabolic subgroup of $G_{\mathbb{C}}$ stabilizing $\mathbb{E}_{k}=\mathbb{F}_{k}$. Let $q_{k}: \check{D}=G_{\mathbb{C}} / Q \longrightarrow \check{D}_{k}:=G_{\mathbb{C}} / Q_{k}$ be the corresponding holomorphic projection of flag varieties. By replacing $E$ by $\Lambda^{r_{k}} E$ (where $r_{k}=\mathrm{rk}^{k}$ ) we can assume that $r_{k}=1$. In this case we obtain the following :

Corollary 1. Let $\rho: \Gamma=\pi_{1}(X) \longrightarrow G$ be the monodromy of a complex variation of Hodge structure $E=\oplus_{i=0}^{k} E^{i}$ on a compact Kähler manifold, with associated period map $f_{\rho}: \tilde{X} \longrightarrow G / V$. Suppose that $r_{k}=r k E^{k}=1$. Then :

- either the $(2,2)$-form ${\overline{f_{\rho}}}^{*} p_{1}(\mathcal{F}) \in A^{2,2}(X, \mathbb{R})$ is non-positive on $X$ and negative at some point of $X$. In this case :

$$
\rho^{*} p_{1}(\mathcal{F}) \neq 0 \in H^{4}(\Gamma, \mathbb{R}) .
$$

- or the map $q_{k} \circ f_{\rho}: \tilde{X} \longrightarrow \check{D}_{k}$ factorizes through a complex curve.

In the second case one easily obtains as in 21] that the pull-back of a well-chosen $G$-invariant real $(1,1)$-form on $\check{D}$ gives a non-trivial element in $H^{2}(\Gamma, \mathbb{R})$, thus finishing the proof of theorem 1 .

1.5. Acknowledgements. It is a pleasure to thank B. Claudon, P.Eyssidieux, V. Koziarz, J.Maubon, C. Simpson and D.Toledo for their interest in this work.

1.6. Organization of the paper. The paper is organized as follows. In section 2 we collect some well-known facts concerning continuous cohomology of real simple Lie groups. In section 3 we justify diagram (10). In section 4 we remind the relevant facts from non-Abelian Hodge theory and in section 5 the geometry of period domains. Section 6 contains the fundamental computation of $\pi^{*} p_{1}(\mathcal{F})$ for pairs of commuting horizontal vectors (c.f. theorem 11) and the proof of theorem 4 . The last two sections prove corollary 1, theorem 1, theorem 2 and theorem 3 ,

\section{Continuous Cohomology of ReAl Simple Lie Groups}

In this section we collect some classical results for the convenience of the reader. For simplicity all the cohomologies will be understood with real coefficients.

2.1. Cartan's theorem. Let $G$ be a connected simple real Lie group of non-compact type, $U$ its compact dual and $K$ its maximal compact subgroup. By Van Est's theorem :

$$
H_{c t}^{\bullet}(G) \simeq H^{\bullet}(\mathfrak{g}, K) \simeq H^{\bullet}(U / K) .
$$

We are thus reduced to compute the cohomology of $U / K$. More generally let $V$ be any connected Lie subgroup of $U$. We want to compute $H^{\bullet}(U / V)$.

First for $V=\{1\}$. Let $\mathfrak{t}_{U}$ and $\mathfrak{t}_{V}, \mathfrak{t}_{V} \subset \mathfrak{t}_{U}$, be maximal Abelian subalgebras of $\mathfrak{u}$ and $\mathfrak{v}$ respectively. By Hopf theorem [3] :

$$
H^{\bullet}(G)=\Lambda^{\bullet}\left(z_{1}, \cdots, z_{n}\right)
$$


where the $z_{i}$ 's are universal transgressive elements. The Cartan-Chevalley theorem implies :

$$
H^{\bullet}(B U)=S^{\bullet}\left(\mathfrak{t}_{U}\right)^{W_{U}}
$$

is the ring of $W_{U}$-invariants polynomials on $\mathfrak{t}_{U}$. If $y_{1}, \cdots, y_{n}$ denotes the image of $z_{1}, \cdots, z_{n}$ by transgression for the $U$-principal bundle $U \hookrightarrow E U \longrightarrow B U$ then the $y_{i}$ 's generate $H^{\bullet}(B U)$.

For any connected $V \subset U$ : let

$$
\rho^{*}(V, U): H^{\bullet}(B U) \simeq S^{*}\left(\mathfrak{t}_{U}\right)^{W_{U}} \longrightarrow S^{*}\left(\mathfrak{t}_{V}\right)^{W_{V}} \simeq H^{\bullet}(B V)
$$

be the natural restriction map. Let $(L, d)$ be the differential algebra defined by :

$$
L=H^{\bullet}(B V) \otimes_{\mathbb{Z}} H^{\bullet}(U)
$$

and

$$
\begin{aligned}
d\left(1 \otimes z_{i}\right) & =\rho^{*}(V, U) y_{i} \otimes 1 \quad(1 \leq i \leq n) \\
d(b \otimes 1) & =0 \quad \text { for } b \in H^{\bullet}(B V) .
\end{aligned}
$$

Let denote by $q: H^{\bullet}(B V) \longrightarrow H^{\bullet}(L, d)$ be the homomorphism induced by the inclusion $\left(H^{\bullet}(B V), 0\right) \subset(L, d)$ of $d g$-algebras and by $\sigma^{*}: H^{\bullet}(B V) \longrightarrow H^{\bullet}(U / V)$ the characteristic homomorphism deduced from the classifying map $U / V \longrightarrow B V$.

Theorem 5 (H.Cartan). [3, p.187] There exists an isomorphism $\mu: H^{\bullet}(L, d) \longrightarrow H^{\bullet}(U / V)$ such that the following diagram commutes :

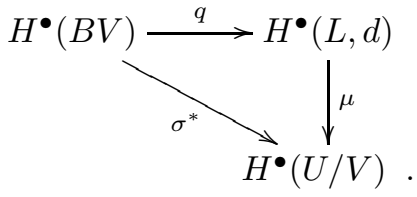

\subsection{Equal rank case.}

Theorem 6. 3, theor 26.1] Let $U / V$ be a compact homogeneous space, with $U$ connected and $V$ connected compact. If $r k U=r k V$ then :

(a) $\rho^{*}(V, U): H^{\bullet}(B U) \longrightarrow H^{\bullet}(B V)$ is injective.

(b) $H^{\bullet}(G / U)=S^{\bullet}\left(\mathfrak{t}_{U}\right)^{W_{V}} /<S^{\bullet}\left(\mathfrak{t}_{U}\right)^{W_{U}}>^{+}$where $\left\langle S^{\bullet}\left(\mathfrak{t}_{U}\right)^{W_{U}}>^{+}\right.$is the ideal of $S^{\bullet}\left(\mathfrak{t}_{U}\right)^{W_{V}}$ generated by elements of $S^{\bullet}\left(\mathfrak{t}_{U}\right)^{W_{U}}$ of positive degrees.

(c) If $s_{i}-1,1 \leq i \leq n$, resp. $r_{j}-1,1 \leq j \leq n$, are the degrees of the $z_{i}$ 's, resp. of universal transgressive elements basis (as an exterior algebra) of $H^{\bullet}(B V)$ then the Poincaré polynomial of $U / V$ is :

$$
P(U / V, t)=\frac{\left(1-t^{s_{1}}\right) \cdots\left(1-t^{s_{l}}\right)}{\left(1-t^{r_{1}}\right) \cdots\left(1-t^{r_{l}}\right)} \quad \text { Hirsch formula) } .
$$

2.3. Low-degree cohomology. Consulting tables for the constants $s_{i}$ and $r_{i}$ [1] one easily deduces from theorem 6 the following :

Corollary 2. Let $U / K$ be a symmetric space of compact type with $U$ connected simple real compact Lie group of dimension larger than 3 . Then :

(a) $h^{2}(U / K)=0$ except if $U$ is of Hermitian type in which case $h^{2}(U / K)=1$.

(b)

$$
h^{4}(U / K)= \begin{cases}0 & \text { if } K \text { is simple } \\ 2 & \text { if } U \text { is of Hermitian type } \\ 1 & \text { otherwise. }\end{cases}
$$


(c) If $r k K=r k U$ then $H^{\bullet}(U / K)$ is concentrated in even degrees, $h^{2}(U / K)=0$ and $h^{4}(U / K)=$ 1 except if $U$ is of Hermitian type in which case $h^{2}(U / K)=1$ and $h^{4}(U / K)=2$.

One can also show by elementary methods :

Theorem 7. Let $U / K$ be a symmetric space of compact type with $U$ connected simple real compact Lie group of dimension larger than 3 and $r k U=$ rkK. Then the first Pontryagin class $\left[p_{1}(\mathcal{F})\right] \in$ $H^{4}(U / K)$ of the real bundle $\mathcal{F}=U \times_{K, \text { Ad }} \mathfrak{k}$ does not vanish.

Remark 3. One also easily shows that if the total Pontryagin class $p(U / K) \in H^{\bullet}(U / K)$ is nontrivial then necessarily $\operatorname{rk} U=\operatorname{rk} K[11]$.

\section{Characteristic Classes of Representations}

Let $\Gamma$ and $G$ be topological groups. Let $\rho: \Gamma \longrightarrow G$ be a group morphism. Our goal in this section is to recall some basic facts concerning the pull-back map it induces in cohomology

$$
\rho^{*}: H_{c}^{\bullet}(G, \mathbb{R}) \longrightarrow H_{c}^{\bullet}(\Gamma, \mathbb{R}),
$$

in particular the commutative diagram (11).

Suppose $\Gamma$ is a discrete group. Thus $\rho$ factorizes through $G^{\delta}$ (the group $G$ with the discrete topology) and the pull-back map in cohomology can be written

$$
H_{c}^{\bullet}(G, \mathbb{R}) \stackrel{i^{*}}{\hookrightarrow} H^{\bullet}\left(G^{\delta}, \mathbb{R}\right)=H^{*}\left(B G^{\delta}, \mathbb{R}\right) \stackrel{\rho^{*}}{\rightarrow} H^{\bullet}(\Gamma, \mathbb{R})
$$

where $i: G^{\delta} \longrightarrow G$ is the canonical map and the injectivity of $i^{*}$ is a classical result of Borel and Selberg.

We will be interested in the case where $\Gamma$ is the fundamental group of a closed smooth manifold and $G$ is a real simple Lie group of non-compact type with maximal compact subgroup $K$ and associated Riemannian symmetric space $G / K$.

3.1. Continuous cohomology versus discrete cohomology for Lie groups. For details on this section we refer to [10]. Let $G$ be as above. The continuous cohomology $H_{c}^{\bullet}(G, \mathbb{R})$ is described by Van Est's theorem :

$$
H_{c}^{\bullet}(G, \mathbb{R}) \simeq H^{\bullet}\left(A^{*}(G / K)^{G}, d\right) \simeq H^{\bullet}(\mathfrak{g}, K, \mathbb{R})
$$

where $\left(A^{*}(G / K)^{G}, d\right)$ denotes the $G$-invariant subcomplex of the real De Rham complex of $G / K$.

Let $\mathcal{F}_{G / K} \longrightarrow B G^{\delta}$ denotes the flat bundle with fiber $G / K$ associated to the canonical flat $G$-bundle $\mathcal{F}_{G}$ over $B G^{\delta}$. As $G / K$ is contractible the bundle $\mathcal{F}_{G / K}$ admits a continuous section $s$, unique up to homotopy. Finally one obtains the following diagram, uniquely defined in the homotopy category :

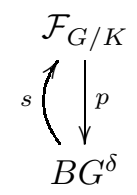

Equivalently the section $s$ can be interpreted as a $G^{\delta}$-equivariant map

$$
E G^{\delta} \stackrel{s}{\longrightarrow} G / K \text {. }
$$

Any $G$-invariant closed form $\lambda \in A^{i}(G / K)$ defines by flat glueing a closed simplicial differential form $\lambda \in A^{i}\left(\mathcal{F}_{G / K}\right)$ with closed pull-back $s^{*} \lambda \in A^{i}\left(B G^{\delta}\right)$. Equivalently $s^{*} \lambda$ is a $G^{\delta}$-invariant closed form on $E G^{\delta}$. 
Finally the map

$$
H_{c}^{\bullet}(G, \mathbb{R}) \stackrel{i^{*}}{\longrightarrow} H^{\bullet}\left(G^{\delta}, \mathbb{R}\right)=H^{*}\left(B G^{\delta}, \mathbb{R}\right)
$$

is canonically identified with :

$$
s^{*}: H^{\bullet}\left(A^{*}(G / K)^{G}, d\right) \simeq H^{\bullet}(\mathfrak{g}, K, \mathbb{R}) \longrightarrow H^{\bullet}\left(G^{\delta}, \mathbb{R}\right)
$$

3.2. The characteristic homomorphism. Let $\Gamma$ be a discrete group and $G$ as above. One has the pull-back diagram, uniquely defined in the homotopy category :

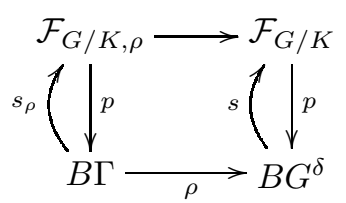

Equivalently the section $s_{\rho}$ can be interpreted as a $\rho$-equivariant continuous map

$$
E \Gamma \stackrel{s_{\rho}}{\longrightarrow} G / K
$$

Suppose moreover that $\Gamma=\pi_{1}(M)$ is the fundamental group of a connected topological space $M$. Gluing the diagram 6 along the classifying map (canonical in the homotopy category) $c: M \longrightarrow B \Gamma$ defined by the universal cover $\tilde{M}$ of $M$, one obtains :

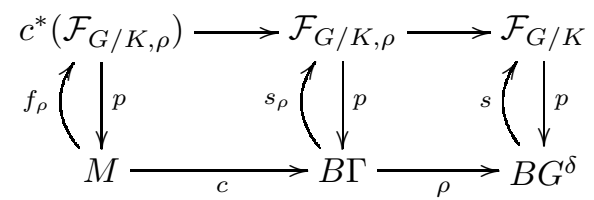

Equivalently the section $f_{\rho}$ can be interpreted as a $\rho$-equivariant continuous map

$$
f_{\rho}: \tilde{M} \stackrel{c}{\longrightarrow} E \Gamma \stackrel{s_{\rho}}{\longrightarrow} G / K
$$

Finally one has the following commutative diagram, which refines diagram (1) :

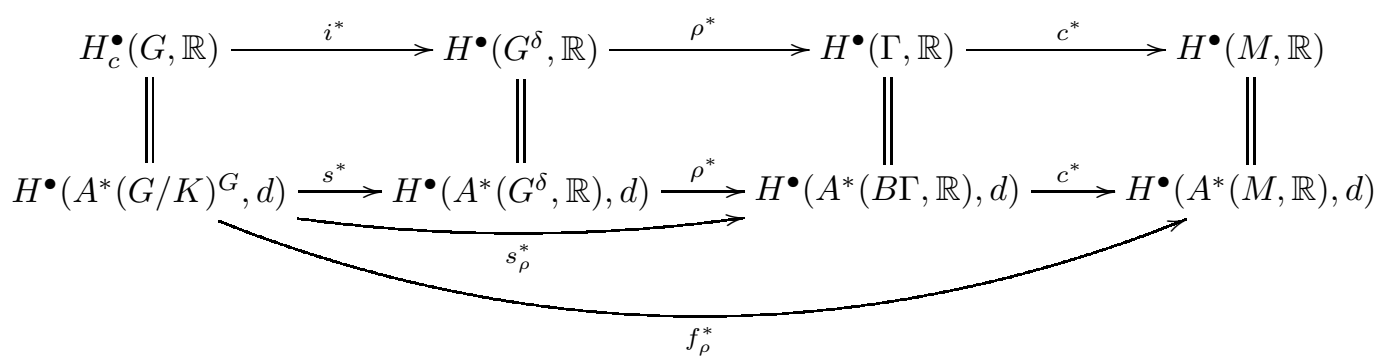

\section{Non-Abelian Hodge theOry}

In this section $\mathbf{G}_{\mathbb{C}}$ is a reductive $\mathbb{C}$-algebraic group and $G_{\mathbb{C}}=\mathbf{G}_{\mathbb{C}}(\mathbb{C})$ its Lie group of complex points. We fix once for all a maximal compact subgroup $U$ of $G_{\mathbb{C}}$. Let $(X, \omega)$ be a compact connected Kähler manifold with fundamental group $\Gamma=\pi_{1}(X)$ (the role of the base point will be unimportant for our discussion). 
4.1. Simpson's correspondence. Non-Abelian Hodge theory establishes a correspondence be-

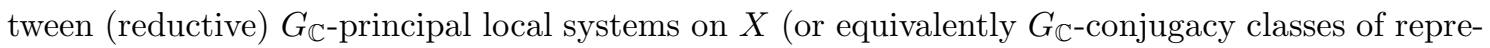
sentations $\Gamma \longrightarrow G_{\mathbb{C}}$ and (semi-harmonic) $\mathbf{G}_{\mathbb{C}}$-principal Higgs bundles on $X$ :

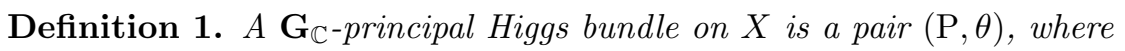

- $\mathrm{P}$ is a principal holomorphic $G_{\mathbb{C}}$-bundle on $X$.

- $\theta \in \operatorname{AdP} \otimes \Omega_{X}^{1}$ satisfies $[\theta, \theta]=0$ (where $\operatorname{AdP}:=\mathrm{P} \times_{G_{\mathbb{C}}} \mathfrak{g}_{\mathbb{C}}$ ).

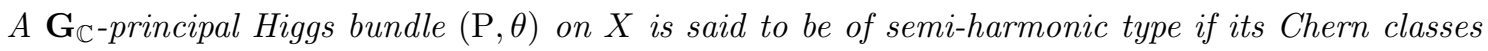
vanish and for some irreducible $\mathbf{G}_{\mathbb{C}}$-module $V$ (and then for any) the Higgs vector-bundle $\mathrm{P} \otimes_{G_{\mathbb{C}}} V$ is Higgs semi-stable.

The correspondence is obtained as follows. Let $\rho: \Gamma \longrightarrow G_{\mathbb{C}}$ be a reductive representation and

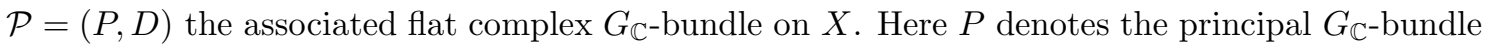
$\tilde{X} \times{ }_{\rho} G_{\mathbb{C}}$ with flat connection $D \in A^{1}(\operatorname{Ad} P)$. As $X$ is compact Kähler and $\rho$ is reductive there exists an essentially unique $\rho$-equivariant harmonic map

$$
f: \tilde{X} \longrightarrow G_{\mathbb{C}} / U
$$

(where $G_{\mathbb{C}} / U$ denotes the symmetric space of $G_{\mathbb{C}}$ ), defining an harmonic $U$-reduction $P_{U}$ of $P$. Decompose the flat connexion $D$ as

$$
D=\nabla+\alpha,
$$

where $\nabla$ is the canonical connexion on the $U$-principal bundle $P_{U}$ and $\alpha \in A^{1}(X, \operatorname{Ad} P)$. Decompose furthermore using types :

$$
\begin{aligned}
& \nabla=\partial_{K}+\bar{\partial} \\
& \alpha=\theta+\theta^{*},
\end{aligned}
$$

where $\partial_{K}$ is of type $(1,0), \bar{\partial}$ is of type $(0,1), \theta \in A^{1,0}(\operatorname{Ad} P)$ and $\theta^{*}=\tau(\theta) \in A^{0,1}(\operatorname{Ad} P)$ is the conjugate of $\theta$ with respect to the $U$-reduction. Define $D^{\prime}=\partial_{K}+\theta^{*}, D^{\prime \prime}=\bar{\partial}+\theta$, thus $D=D^{\prime}+D^{\prime \prime}$. As $D$ is flat and the $U$-reduction $P_{U}$ is harmonic, $\left(D^{\prime \prime}\right)^{2}=0$, that is :

$$
\bar{\partial}^{2}=\bar{\partial}(\theta)=[\theta, \theta]=0
$$

Finally $(\mathrm{P}=(P, \bar{\partial}), \theta)$ is a $\mathbf{G}_{\mathbb{C}^{-H i g g s}}$ bundle (of semi-harmonic type). Notice that knowing $(\mathrm{P}, \theta)$ is equivalent to knowing $\left(P, D^{\prime \prime}\right)$.

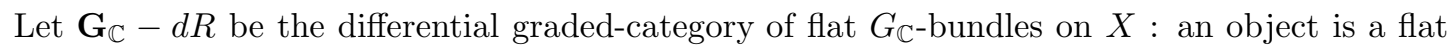
bundle $\mathcal{P}=(P, D)$ on $X$ and

$$
\operatorname{Hom}_{\mathbf{G}_{\mathbb{C}}-d R}\left(\mathcal{P}, \mathcal{P}^{\prime}\right)=\left(A^{\bullet}\left(\operatorname{Hom}\left(\operatorname{Ad} P, \operatorname{Ad} P^{\prime}\right), D_{\operatorname{Hom}\left(P, P^{\prime}\right)}\right) .\right.
$$

Let $\mathbf{G}_{\mathbb{C}}-$ Dol be the differential graded-category of semi-harmonic $\mathbf{G}_{\mathbb{C}}$-Higgs bundles on $X:$ an object is a semi-harmonic $\mathbf{G}_{\mathbb{C}}$-Higgs bundle $\left(\mathrm{P}, \theta_{\mathrm{P}}\right)$ on $X$ and

$$
\left.\operatorname{Hom}_{\mathbf{G}_{\mathbb{C}}-D o l}\left(\mathrm{P}, \theta_{\mathrm{P}}\right),\left(\mathrm{P}^{\prime}, \theta_{\mathrm{P}^{\prime}}\right)\right)=\left(A^{\bullet}\left(\operatorname{Hom}\left(\operatorname{Ad} P, \operatorname{Ad} \mathcal{P}^{\prime}\right), D_{\operatorname{Hom}\left(P, P^{\prime}\right)}^{\prime \prime}\right) .\right.
$$

Theorem 8 (Simpson). The functor $F: \mathbf{G}_{\mathbb{C}}-d R \longrightarrow \mathbf{G}_{\mathbb{C}}-$ Dol associating to the flat bundle $\mathcal{P}$ the Higgs bundle $(\mathrm{P}, \theta)$ is a quasi-equivalence of differential graded categories.

It implies the geometric (weaker) version [28, theor. 9.11 and lemma 9.14] :

Theorem 9 (Simpson). The functor $F$ induces a real-analytic diffeomorphism

$$
\phi_{\mathbf{G}}: \mathbf{M}\left(\Gamma, \mathbf{G}_{\mathbb{C}}\right)(\mathbb{C}) \longrightarrow \mathbf{M}_{\mathrm{Dol}}\left(X, \mathbf{G}_{\mathbb{C}}\right)(\mathbb{C})
$$


between the Betti moduli space $\mathbf{M}(\Gamma, \mathbf{G})(\mathbb{C})=\left(\operatorname{Hom}\left(\Gamma, \mathbf{G}_{\mathbb{C}}\right) / / \mathbf{G}_{\mathbb{C}}^{\text {ad }}\right)(\mathbb{C})$ of representations of $\Gamma=$ $\pi_{1}(X)$ in $G_{\mathbb{C}}$ and the Dolbeault moduli space of $\mathbf{G}_{\mathbb{C}}$-Higgs bundles of semi-harmonic type.

4.2. G-variations of Hodge structures. The moduli space $\mathbf{M}_{\mathrm{Dol}}(X, \mathbf{G})$ of $\mathbf{G}_{\mathbb{C}}$-Higgs bundles of semi-harmonic type carries a natural $\mathbb{C}^{*}$-action : an element $t \in \mathbb{C}^{*}$ maps $[(\mathrm{P}, \theta)]$ to $[(\mathrm{P}, t \cdot \theta)]$ [28, p.62]. The fixed points of this action are of particular importance : they are systems of $\mathbf{G}-$ Hodge bundles [27, p.44] and correspond by Simpson's correspondence to (isomorphism classes of) G-complex variations of Hodge structure (G-CVHS).

\subsubsection{Hodge datum.}

Definition 2. A (pointed) Hodge datum is a pair $(\mathbf{G}, u)$, where $\mathbf{G}$ is a real reductive algebraic group and $u: \mathbf{U}(1) \longrightarrow \operatorname{Aut}(\mathbf{G})^{0} \subset \mathbf{G}^{\text {ad }}$ is a morphism of real algebraic groups such that $C=u(-1)$ is a Cartan involution of $\mathbf{G}$ (that is : $C^{2}=1$ and $\tau:=C \sigma=\sigma C$ is the conjugation of $\mathbf{G}_{\mathbb{C}}$ with respect to a compact real form $\mathbf{U}$, where $\sigma$ denotes the conjugation of $\mathbf{G}_{\mathbb{C}}$ with respect to $\left.\mathbf{G}\right)$.

In particular the Cartan involution $C$ is inner. One easily shows (c.f. [27, section 4.4]) that an algebraically connected real reductive group $\mathbf{G}$ admits a $\operatorname{Hodge} \operatorname{datum}(\mathbf{G}, u)$ (one says that $\mathbf{G}$ is of Hodge type) if and only if $\mathbf{G}$ contains an anisotropic maximal torus $\mathbf{T}$. In other words the reductive real Lie group $\mathbf{G}(\mathbb{R})$ has the same real rank than any of its maximal compact subgroups.

\subsubsection{Period domains.}

Definition 3. Let $(\mathbf{G}, u)$ be a Hodge datum. We denote by :

- $V=Z_{G}(u)$ the centralizer of $u$ in $G=\mathbf{G}(\mathbb{R})$. As $V$ is invariant by $C, V$ is contained in $U=\mathbf{U}(\mathbb{R})$, in particular $V$ is compact.

- $K$ the centralizer of $C$ in $L$. Notice that $K$ coincide with the intersection $U \cap L$. Thus the group $K$ is a maximal compact subgroup of $L$.

Finally the Hodge datum $(\mathbf{G}, u)$ defines canonically the chain of inclusions of compact groups $V \subset K \subset U$.

Let $(\mathbf{G}, u)$ be a Hodge datum. Let $\lambda: \mathbf{G} \longrightarrow \mathbf{G L}(E)$ be a real (resp. complex) representation $\lambda: \mathbf{G} \longrightarrow \mathbf{G L}(E)$ of $\mathbf{G}$. If $\mathbf{G}=\mathbf{G}^{\text {ad }}$ (or more generally if $\lambda$ factorizes through $\mathbf{G}^{\text {ad }}$ ) the composite $\lambda \circ u: \mathbf{U}(1) \longrightarrow \mathbf{G}^{\text {ad }} \longrightarrow \mathbf{G L}(E)$ defines a weight 0 real (resp. complex) Hodge structure on $E$ polarized by $\lambda \circ u(-1)$. In particular the adjoint representation of $\mathbf{G}$ defines on the Lie algebra $\mathfrak{g}$ a weight 0 polarized real Hodge structure :

$$
\mathfrak{g}_{\mathbb{C}}=\bigoplus_{i \in \mathbb{Z}} \mathfrak{g}_{\mathbb{C}}^{i,-i}
$$

where $u(z)$ acts on $\mathfrak{g}_{\mathbb{C}}^{i,-i}$ via multiplication by $z^{-i}$. We will denote by $F^{\bullet} \mathfrak{g}_{\mathbb{C}}$ the corresponding decreasing Hodge filtration. The polarization is given by the Killing form $\beta_{\mathbf{G}}$.

Definition 4. One denotes by $\mathfrak{q} \subset \mathfrak{g}_{\mathbb{C}}$ the Lie sub-algebra $F^{0} \mathfrak{g}_{\mathbb{C}}$ and $Q \subset G_{\mathbb{C}}$ the corresponding subgroup.

One easily check that $\mathfrak{q}$ is a parabolic sub-algebra of $\mathfrak{g}_{\mathbb{C}}$, with Levi sub-algebra $\mathfrak{v}_{\mathbb{C}}$ the complexified Lie algebra of $\mathfrak{v}=\operatorname{Lie}(V)$.

Definition 5. Let $(\mathbf{G}, u)$ be a Hodge datum. The period domain $D$ associated to $(\mathbf{G}, u)$ is the G-conjugacy class of $u$. 
Thus $D$ naturally identifies with $G / V$. Let $\check{D}=G_{\mathbb{C}} / Q$ be the flag manifold of $G_{\mathbb{C}}=\mathbf{G}(\mathbb{C})$ defined by $Q$, the natural morphism $D=G / V \hookrightarrow \check{D}=G_{\mathbb{C}} / Q$ is an open embedding and thus defines a natural $G$-invariant complex structure on $D$.

4.2.3. Horizontality. The holomorphic tangent bundle $T D$ naturally identifies with the $G$-equivariant bundle $\left(G_{\mathbb{C}} \times_{Q} \mathfrak{g}_{\mathbb{C}} / \mathfrak{q}\right)_{\mid D}$.

Definition 6. The superhorizontal tangent bundle $T_{s h} D$ is the holomorphic sub-bundle $\left(G_{\mathbb{C}} \times_{Q}\right.$ $\left.F^{-1} \mathfrak{g}_{\mathbb{C}} / \mathfrak{q}\right)_{\mid D}$ of $T D$.

Definition 7. Let $\mathcal{C}$ be the category whose objects are pairs $\left(Y, R_{Y}\right)$, where $Y$ is a complex smooth analytic space, $R_{Y} \subset T Y$ a holomorphic distribution, and a morphism $f:\left(Y, R_{Y}\right) \longrightarrow\left(X, R_{X}\right)$ in $\mathcal{C}$ is a holomorphic horizontal map $f: X \longrightarrow Y$ : one requires that $d f\left(R_{Y}\right) \subset R_{X}$. We will look at the category of smooth analytic spaces as a subcategory of $\mathcal{C}$, the distribution being the full tangent space.

4.2.4. $\mathbf{G}_{\mathbb{C}}-\mathbb{C} V H S$. With all these definitions we can define the main actors in Simpson's theory:

Definition 8. Let $X$ be a complex analytic manifold with fundamental group $\Gamma$ and universal cover $\tilde{X}$. Let $\mathbf{G}_{\mathbb{C}}$ be a complex reductive algebraic group. A $\mathbf{G}_{\mathbb{C}}$-complex variation of Hodge structure $\left(\mathbf{G}_{\mathbb{C}}-\mathbb{C} V H S\right)$ is a Hodge datum $(\mathbf{G}, u)$ with period domain $D$ for a real form $\mathbf{G}$ of $\mathbf{G}_{\mathbb{C}}$, a representation $\rho: \Gamma \longrightarrow G=\mathbf{G}(\mathbb{R}) \subset G$ (called the monodromy of the variation) and a holomorphic horizontal $\rho$-equivariant map $f: \tilde{X} \longrightarrow D$ (called period map).

Definition 9. Let $(\mathbf{G}, u, \rho, f: \tilde{X} \longrightarrow D)$ be $a \mathbf{G}_{\mathbb{C}}-\mathbb{C} V H S$ on $X$. Let $\tau: Q \longrightarrow V_{\mathbb{C}} \stackrel{i}{\hookrightarrow} G_{\mathbb{C}}$ be the reduction of $i$ to the Levi $V_{\mathbb{C}}$ of $Q$. One associates to it the following principal $G_{\mathbb{C}}$-bundles on $X$ :

- the flat $G_{\mathbb{C}}$-bundle $\mathcal{P}=\tilde{X} \times_{\Gamma, \rho} G_{\mathbb{C}}$, which is naturally a holomorphic bundle. Notice that this holomorphic structure is compatible with the identification $\mathcal{P}:=f^{*}\left(\left(L_{\mathbb{C}} \times_{Q, i} G_{\mathbb{C}}\right)_{\mid D}\right)$ (descent to $X$ of the) pull-back via $f$ of the holomorphic $G_{\mathbb{C}}$-bundle $G_{\mathbb{C}} \times_{Q, i} G_{\mathbb{C}}$ on $\check{D}$.

- the holomorphic $G_{\mathbb{C}}$-bundle $\mathrm{P}=f^{*}\left(\left(L_{\mathbb{C}} \times_{Q, \tau} G_{\mathbb{C}}\right)_{\mid D}\right.$ (descent to $X$ of the) pull-back via $f$

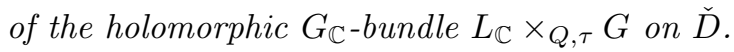

4.3. G-CVHS and Simpson correspondence. Let $\left(\mathbf{G}, u: \mathbf{U}(1) \longrightarrow \mathbf{G}^{\text {ad }}, \rho: \Gamma \longrightarrow G, f:\right.$ $\tilde{X} \longrightarrow D$ ) be a $\mathbf{G}_{\mathbb{C}}-\mathbb{C V H S}$ on $X$.

On the Betti side it canonically defines the isomorphism class of the flat $G$-bundle $\mathcal{P}$.

On the Dolbeault side : the adjoint bundle AdP $=\mathrm{P} \times_{G_{\mathbb{C}}, \text { Ad }} \mathfrak{g}_{\mathbb{C}}=f^{*}\left(\left(G_{\mathbb{C}} \times_{Q, \text { Ado } \tau} \mathfrak{g}_{\mathbb{C}}\right)_{\mid D}\right)$ identifies with the graded bundle $\operatorname{Gr}_{F} \operatorname{Ad} \mathcal{P}$ of the weight 0 complex variation of Hodge structure $\operatorname{Ad} \mathcal{P}=\mathcal{E}_{\mathfrak{g}}$ associated to the representation $\mathbf{G}_{\mathbb{C}} \longrightarrow \mathbf{G L}\left(\mathfrak{g}_{\mathbb{C}}\right)$ :

$$
\mathrm{AdP}=\bigoplus_{p \in \mathbb{Z}}(\mathrm{AdP})^{p,-p}
$$

Definition 10. Define $\theta_{f} \in(\mathrm{AdP})^{-1,1} \otimes \Omega_{X}^{1}$ as the differential of $f$.

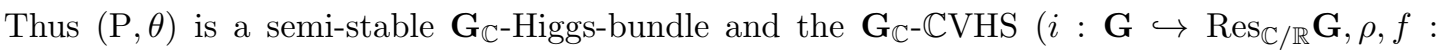
$\tilde{X} \longrightarrow D$ ) canonically defines the point $[(\mathrm{P}, \theta)]$ (called a system of $\mathbf{G}$-Hodge bundles by Simpson) in $\mathbf{M}_{\mathrm{Dol}}(X, \mathbf{G})$.

Proposition 1. 27, cor 4.2] Let $[\rho] \in \mathbf{M}\left(\Gamma, \mathbf{G}_{\mathbb{C}}\right)(\mathbb{C})$ with $\rho$ reductive. Then $\phi_{\mathbf{G}_{\mathbb{C}}}([\rho]) \in \mathbf{M}_{\text {Dol }}\left(X, \mathbf{G}_{\mathbb{C}}\right)(\mathbb{C})$ is $\mathbb{C}^{*}$-fixed if and only if $\rho$ is the monodromy of a $\mathbf{G}_{\mathbb{C}}$-complex variation of Hodge structure $f: \tilde{X} \longrightarrow D$. Moreover $\phi_{\mathbf{G}_{\mathbb{C}}}([\rho])=[(\mathrm{P}, \theta)]$. 


\section{Geometry of Period domains}

5.1. The fibration $D=G / V \longrightarrow G / K$. The action of the compact form $U \subset G_{\mathbb{C}}$ on $\check{D}$ is transitive with stabilizer $V$ at the point $u \in \check{D}$. The inclusion $V \subset K \subset G$ (resp. $V \subset K \subset U$ ) defines a $G$-equivariant (resp. $U$-equivariant) $\mathcal{C}^{\infty}$ fibration $\pi: D=G / V \longrightarrow G / K$ (resp. $\check{\pi}: \check{D}=$ $U / V \longrightarrow U / K)$ over the symmetric space $G / K$ (resp. its dual $U / K$ ) :

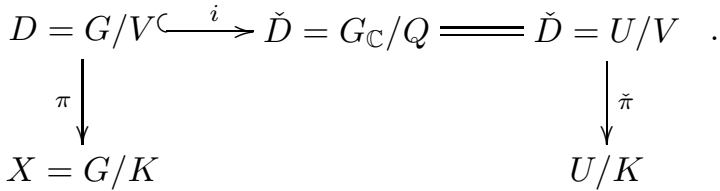

The symmetric space $G / K$ (resp. $U / K$ ) does not admit any $G$-invariant (resp. $U$-invariant) complex structure except in the case where $G$ is of Hermitian type (i.e. when the Hodge structure on $\mathfrak{g}$ is of type $(-1,1),(0,0)$ and $(1,-1))$. Even when $G$ is of Hermitian type the projections $\pi: D \longrightarrow G / K$ and $\check{\pi}: \check{D} \longrightarrow U / K$ are not holomorphic in general. However their fibers (in particular the fibre $K / V$ over the base point $u \in D$ ) are always compact holomorphic subvarieties of $D$ and $\check{D}$ [14, p.261]. In particular $D$ is a Stein manifold only in the case where $D=X$ is a Hermitian symmetric space. Let us also emphasize that the projections $\pi: D \longrightarrow G / K$ and $\check{\pi}_{\mid D}: D \longrightarrow U / K$ do not coïncide (even if they have the same fibre $K / V$ at the base point $u \in D$ ).

5.2. Root data. Let $(\mathbf{G}, u)$ be a Hodge datum with Cartan involution $C$. Let $V \subset K \subset U$ be the compact subgroups of $G_{\mathbb{C}}$ it defines (c.f. section 4.2.1). We will also denote by $\sigma$ and $\tau$ the complex conjugations of $\mathfrak{g}_{\mathbb{C}}$ with respect to $\mathfrak{g}$ and $\mathfrak{u}$ respectively. Let $T \subset V$ be a maximal compact torus of $G$ containing $u(U(1))$. Let $\mathfrak{t}$ be its Lie algebra and $\mathfrak{h}_{\mathbb{C}}=\mathfrak{t} \otimes \mathbb{C}$ be the corresponding Cartan subalgebra of $\mathfrak{g}_{\mathbb{C}}$. The Cartan subalgebra $\mathfrak{h}_{\mathbb{C}}$ is $\tau$-stable and $\sigma$-stable.

The adjoint representation of $\mathfrak{h}_{\mathbb{C}}$ on $\mathfrak{g}_{\mathbb{C}}$ defines a root space decomposition

$$
\mathfrak{g}_{\mathbb{C}}=\mathfrak{h}_{\mathbb{C}} \oplus \bigoplus_{\alpha \in \Delta} \mathfrak{g}_{\alpha}
$$

where $\Delta=\Delta\left(\mathfrak{g}_{\mathbb{C}}, \mathfrak{h}_{\mathbb{C}}\right) \subset \mathfrak{h}_{\mathbb{C}}^{*}$ denotes the root system of $\mathfrak{h}_{\mathbb{C}}$ in $G_{\mathbb{C}}$ and each root space

$$
\mathfrak{g}_{\alpha}=\left\{x \in \mathfrak{g}_{\mathbb{C}} /[h, x]=\alpha(h) x \text { pour tout } h \in \mathfrak{h}_{\mathbb{C}}\right\}
$$

is of complex dimension 1. Let $\Delta\left(\mathfrak{v}_{\mathbb{C}}, \mathfrak{h}_{\mathbb{C}}\right) \subset \Delta\left(\mathfrak{k}_{\mathbb{C}}, \mathfrak{h}_{\mathbb{C}}\right) \subset \Delta$ be the root subsystems associated to $\left(\mathfrak{v}_{\mathbb{C}}, \mathfrak{h}_{\mathbb{C}}\right)$ and $\left(\mathfrak{k}_{\mathbb{C}}, \mathfrak{h}_{\mathbb{C}}\right)$ respectively. We denote by $<\cdot, \cdot>$ the natural scalar product on $\mathfrak{h}_{\mathbb{C}}^{*}$.

Let $\mathfrak{g}=\mathfrak{k} \oplus \mathfrak{p}$ be the Cartan decomposition of $\mathfrak{g}$ defined by the Cartan involution $C$. The involution $C$ commutes with the adjoint action of $\mathfrak{h}_{\mathbb{C}}$ thus any $\mathfrak{g}_{\alpha}, \alpha \in \Delta$ is either contained in $\mathfrak{k}_{\mathbb{C}}$ (the root $\alpha$ is said to be compact) or in $\mathfrak{p}_{\mathbb{C}}$ (the root $\alpha$ is said non-compact). We denote by $\Delta_{\mathfrak{k}} \subset \Delta$ (resp. $\Delta_{\mathfrak{p}} \subset \Delta$ ), the set of compact roots (resp. non-compact roots).

Let $\Delta_{+} \subset \Delta$ be the unique set of positive roots such that $\mathfrak{g}_{-\alpha} \subset \mathfrak{q}$ for every $\alpha \in \Delta_{+}$. Let $\Psi=\left\{\alpha_{1}, \cdots, \alpha_{r}\right\}$ be the corresponding set of simple roots. For every subset $\theta \subset \Psi$ we denote by :

- $|\theta|$ the cardinal of $\theta$.

- $\theta^{c}:=\Psi \backslash \theta$ and $\langle\theta\rangle$ the set of roots in $\Delta_{+}$linear combination of elements of $\theta$.

- $\mathfrak{n}_{\theta,-}$ the nilpotent Lie algebra $\bigoplus_{\alpha \in \Delta_{+} \backslash<\theta>} \mathfrak{g}_{-\alpha}$. 
- $\mathfrak{l}_{\theta}$ the reductive subalgebra centralizer of $\cap_{\alpha \in \theta}$ ker $\alpha$, thus

$$
\mathfrak{l}_{\theta}=\mathfrak{h}_{\mathbb{C}} \oplus \bigoplus_{\alpha \in<\theta>}\left(\mathfrak{g}_{-\alpha} \oplus \mathfrak{g}_{\alpha}\right)
$$

- $\mathfrak{q}_{\theta}$ the parabolic subalgebra $\mathfrak{l}_{\theta} \oplus \mathfrak{n}_{\theta,-}$ and $\mathbf{Q}_{\theta} \subset \mathbf{G}_{\mathbb{C}}$ the corresponding parabolic subgroup.

Remark 4. We followed the convention that $\mathbf{Q}_{\theta}$ is of parabolic rank $\left|\theta^{c}\right|$. Thus $Q_{\Psi}=G_{\mathbb{C}}$ and $Q_{\emptyset}=B$ is the standard Borel subgroup of $G_{\mathbb{C}}$ associated to $\Delta_{+}$.

For $\theta \subset \Psi$ we denote by $\check{D}_{\theta}$ the flag variety $\check{D}_{\theta}=G_{\mathbb{C}} / Q_{\theta}$. For $\theta \subset \theta^{\prime} \subset \Psi$ let $\pi_{\theta \theta^{\prime}}: \check{D}_{\theta} \longrightarrow \check{D}_{\theta^{\prime}}$ be the natural holomorphic projection.

As the subalgebra $\mathfrak{v}_{\mathbb{C}}$ is reductive and $\mathfrak{h}_{\mathbb{C}} \subset \mathfrak{v}_{\mathbb{C}} \subset \mathfrak{k}_{\mathbb{C}}$ there exists a unique subset $\Phi \subset \Delta_{k} \cap \Psi$ such that

$$
\mathfrak{v}_{\mathbb{C}}=\mathfrak{l}_{\Phi} \quad \text { and } \quad \mathfrak{q}=\mathfrak{q}_{\Phi}
$$

The group $U$ is a compact form of $G_{\mathbb{C}}$ thus the roots $\alpha \in \Delta$ are real on $\mathfrak{h}_{\mathbb{R}}:=\sqrt{-1} \mathfrak{t} \subset \mathfrak{h}_{\mathbb{C}}$. This identifies $\Delta$ with a subset of the dual space $\mathfrak{h}_{\mathbb{R}}^{*}$. The root system $\Delta$ cuts $\mathfrak{h}_{\mathbb{R}}$ (and also using the Killing form the dual space $\mathfrak{h}_{\mathbb{R}}^{*}$ ) in a disjoint union of facets. The open facets are the Weyl chambers. Let $\mathfrak{h}_{\mathbb{R},+}$ be the Weyl chamber of $\mathfrak{h}_{\mathbb{R}}$ defined by $\Psi$. Let $E \in \mathfrak{t}$ be the generator of $u: U(1) \longrightarrow G$. The subset $\Phi \in \Psi$ naturally identifies with the set of roots $\alpha$ satisfying $\alpha(\sqrt{-1} E) \geq 0$.

Let $W$ be the Weyl group of $\Delta, s_{\alpha} \in W$ be the reflection associated to $\alpha$ and $w_{0}$ be the element of $W$ of maximal length.

\subsection{Automorphic bundles.}

Definition 11. Let $\pi: Q_{\Phi} \longrightarrow G L(E)$ be a finite dimensional complex representation. We denote by $\check{\mathcal{F}}_{\pi}:=G_{\mathbb{C}} \times_{Q_{\theta}, \pi} E$ the holomorphic $G_{\mathbb{C}}$-equivariant bundle on $\check{D}$ with fibre $E$ associated to $\pi$ and by $\mathcal{F}_{\pi}$ its restriction to $D$.

Let $\mathfrak{n}_{-}=\mathfrak{n}_{\Phi,-}$ be the nilpotent radical of the parabolic subalgebra $\mathfrak{q}=\mathfrak{q}_{\Phi}$. Then

$$
\mathfrak{n}_{+}:=\sigma\left(\mathfrak{n}_{-}\right)=\tau\left(\mathfrak{n}_{-}\right)=F^{-\infty} \mathfrak{g}_{\mathbb{C}} / F^{0} \mathfrak{g}_{\mathbb{C}}=\oplus_{\alpha \in \Delta_{+} \backslash<\Phi>\mathfrak{g}_{\alpha}} .
$$

The complex vector space $\mathfrak{n}_{+}$is a natural $\mathfrak{q}$-module and $T \check{D}=\check{\mathcal{F}}_{\mathfrak{n}_{+}}, T D=\mathcal{F}_{\mathfrak{n}_{+}}$.

Definition 12. We will denote by $\Delta^{-1,1} \subset \Delta_{+}$the unique set of positive roots such that

$$
\mathfrak{g}_{\mathbb{C}}^{-1,1}=\bigoplus_{\alpha \in \Delta^{-1,1}} \mathfrak{g}_{\alpha}
$$

For convenience we will put a total order $<_{t}$ on $\Delta^{-1,1}$ (we will distinguish it from the partial order $\leq$ on $\Delta$ defined by $\Psi)$.

Remark 5. The set $\Delta^{-1,1}$ admits a natural decomposition corresponding to a decomposition of the sub-q-module $\mathfrak{g}_{\mathbb{C}}^{-1,1}=F^{-1} \mathfrak{g}_{\mathbb{C}} / F^{0} \mathfrak{g}_{\mathbb{C}}$ into a sum of blocs :

$$
\mathfrak{g}_{\mathbb{C}}^{-1,1}=\bigoplus_{\beta \in \Phi^{c}} \mathfrak{g}_{\mathbb{C}, \beta}^{-1,1},
$$

with

$$
\mathfrak{g}_{\mathbb{C}, \beta}^{-1,1}:=\bigoplus_{\gamma \in(\beta+<\theta>) \cap \Delta_{+}} \mathfrak{g}_{\gamma} .
$$


Correspondingly :

$$
T_{s h} D=\mathcal{F}_{\mathfrak{g}_{\mathbb{C}}^{-1,1}}=\bigoplus_{\beta \in \Phi^{c}} \mathcal{F}_{\mathfrak{g}_{\mathbb{C}, \beta}^{-1,1}}
$$

which corresponds to the usual Hodge bloc decomposition $\bigoplus \operatorname{Hom}\left(H^{p, q}, H^{p-1, q+1}\right)$.

5.4. Curvature of automorphic bundles. As a complex bundle the holomorphic fiber bundle $\mathcal{F}_{\pi}=\check{\mathcal{F}}_{\pi}$ can be written $G \times_{V, \pi_{\mid V}} E$. As the group $V$ is compact on can put on $E$ a $V$-invariant Hermitian metric which induces a $G$-invariant Hermitian metric on $\mathcal{F}_{\pi}$. The Chern connexion on the Hermitian holomorphic bundle $\mathcal{F}_{\pi}$ is $G$-invariant and is deduced from the connexion form of the $V$-principal bundle $V \hookrightarrow G \longrightarrow G / V=D$. We will denote by $\Theta_{D}(\pi) \in A^{1,1}(D) \otimes \operatorname{End}\left(\mathcal{F}_{\pi}\right)$ its $G$-invariant curvature form. These curvature forms are computed in [14].

Lemma 1. 14, p.265] There exists a basis $\left(e_{\alpha} \in \mathfrak{g}_{\alpha}, h_{\alpha} \in \mathfrak{h}_{\mathbb{R}}\right)_{\alpha \in \Delta}$ of $\mathfrak{g}_{\mathbb{C}}$ (called a Weyl basis) satisfying the following properties :

(a) $\left(e_{\alpha}, e_{\beta}\right)=\delta_{\alpha,-\beta},\left[e_{\alpha}, e_{-\alpha}\right]=h_{\alpha}$.

(b) $\left(h_{\alpha}, x\right)=\alpha(x)$ for $x \in \mathfrak{h}_{\mathbb{C}}$.

(c) $\left[e_{\alpha}, e_{\beta}\right]=0$ if $\alpha \neq-\beta$ et $\alpha+\beta \notin \Delta$.

(d) $\left[e_{\alpha}, e_{\beta}\right]=N_{\alpha, \beta} e_{\alpha+\beta}$ si $\alpha, \beta, \alpha+\beta \in \Delta$, where $N_{\alpha, \beta} \in \mathbb{R}, N_{-\alpha,-\beta}=-N_{\alpha, \beta}$.

(e) $\tau\left(e_{\alpha}\right)=-e_{\alpha}$.

(f) $\sigma(\alpha)=\varepsilon_{\alpha} e_{-\alpha}$ where $\varepsilon_{\alpha}=-1$ if $\alpha$ is compact and $\varepsilon_{\alpha}=1$ if $\alpha$ is non-compact.

(g) $\varepsilon_{\alpha+\beta}=-\varepsilon_{\alpha} \varepsilon_{\beta}$ if $\alpha, \beta, \alpha+\beta \in \Delta$.

Definition 13. Let $\left(e_{\alpha} \in \mathfrak{g}_{\alpha}, h_{\alpha} \in \mathfrak{h}_{\mathbb{R}}\right)_{\alpha \in \Delta}$ be a Weyl basis. We denote by $w_{\alpha} \in \mathfrak{g}_{\alpha}^{*}, \alpha \in \Delta$, the linear form dual to $e_{\alpha}$.

Theorem 10. [14, p.269]

(1) The curvature form $\Theta_{D}$ for the natural connection on the $V$-principal bundle $V \hookrightarrow G \longrightarrow$ $G / V=D$ is

$$
\Theta_{D}=-\sum_{\alpha, \beta \in \Delta^{+} \backslash \Delta_{\mathfrak{t}_{\mathbb{C}}}}\left[e_{\alpha}, e_{-\beta}\right]_{\mathfrak{v}_{\mathbb{C}}} \otimes w_{\alpha} \wedge \overline{w_{\beta}}+\sum_{\alpha, \beta \in\left(\Delta\left(\mathfrak{k}_{\mathbb{C}}\right) \cap \Delta_{+}\right) \backslash<\Phi>}\left[e_{\alpha}, e_{-\beta}\right]_{\mathfrak{v}_{\mathbb{C}}} \otimes w_{\alpha} \wedge \overline{w_{\beta}} .
$$

(2) Let $\pi: Q_{\Phi} \longrightarrow G L(E)$ be a finite dimensional complex representation. The curvature form $\Theta_{D}(\pi) \in A^{1,1}(D) \otimes \operatorname{End} \mathcal{F}_{\pi}$ for the natural Hermitian connection on the automorphic vector bundle $\mathcal{F}_{\pi}$ is

$$
\Theta_{D}(\pi)=-\sum_{\alpha, \beta \in \Delta^{+} \backslash \Delta_{\mathfrak{t}_{\mathbb{C}}}} \pi\left(\left[e_{\alpha}, e_{-\beta}\right]_{\mathfrak{v}_{\mathbb{C}}}\right) \otimes w_{\alpha} \wedge \overline{w_{\beta}}+\sum_{\alpha, \beta \in\left(\Delta\left(\mathfrak{k}_{\mathbb{C}}\right) \cap \Delta_{+}\right) \backslash<\Phi>} \pi\left(\left[e_{\alpha}, e_{-\beta}\right]_{\mathfrak{v}_{\mathbb{C}}}\right) \otimes w_{\alpha} \wedge \overline{w_{\beta}} .
$$

Corollary 3. Let $\pi: Q_{\Phi} \longrightarrow G L(E)$ be a finite dimensional complex representation. Let $\xi \in$ $T_{s h} D \simeq \mathfrak{g}_{\mathbb{C}}^{-1,1}$ be a superhorizontal holomorphic vector field. Then

$$
\Theta_{D}(\pi)(\xi \wedge \bar{\xi})=-[\xi, \sigma(\xi)] \in \mathfrak{v}_{\mathbb{C}}=\mathfrak{g}_{\mathbb{C}}^{0,0} .
$$

\section{First Pontryagin Class : Proof of theorem 4}

6.1. Computation of $\pi^{*} p_{1}(\mathcal{F})$. Let $\pi: D=G / V \longrightarrow G / K$ be the canonical projection. Let

$$
\mathcal{F}:=G \times_{K, \text { Ad }} \mathfrak{k}
$$

be the real oriented $\mathcal{C}^{\infty}$ vector bundle on $G / K$ associated to the $K$-principal bundle $K \hookrightarrow G \longrightarrow$ $G / K$ and the adjoint representation $\mathrm{Ad}: K \longrightarrow \operatorname{Aut}(\mathfrak{k})$. We denote by $p_{1}(\mathcal{F}) \in H^{4}(\mathfrak{g}, K, \mathbb{R})$ the first Pontryagin form of the bundle $\mathcal{F}$ with its canonical metric associated to the $K$-invariant scalar 
product $-B_{\mathfrak{k}}(X, Y)$. By definition $p_{1}(\mathcal{F})=-c_{2}\left(\mathcal{F} \otimes_{\mathbb{R}} \mathbb{C}\right)$ the second Chern form of the complexified bundle $\mathcal{F} \otimes_{\mathbb{R}} \mathbb{C}$. Notice that the pull-back complex bundle $\pi^{*} \mathcal{F} \otimes_{\mathbb{R}} \mathbb{C}$ with its pulled-back Hermitian metric is in fact an automorphic bundle on $G / V$ :

$$
\pi^{*} \mathcal{F} \otimes_{\mathbb{R}} \mathbb{C}=\mathcal{F}_{\mathfrak{E}_{\mathbb{C}}} .
$$

Thus

$$
\pi^{*} p_{1}(\mathcal{F})=p_{1}\left(\mathcal{F}_{\mathfrak{k}}\right)=-c_{2}\left(\mathcal{F}_{\mathfrak{k}_{\mathbb{C}}}\right) \in H^{4}(\mathfrak{g}, V, \mathbb{R})
$$

lies in $H^{2,2}(\mathfrak{g}, V, \mathbb{R})$.

Theorem 11. Let $\xi, \eta \in \mathfrak{g}_{\mathbb{C}}^{-1,1}$ satisfying $[\xi, \eta]=0$. Then

$$
-8 \pi^{2} \cdot \pi^{*} p_{1}(\mathcal{F})(\xi \wedge \eta \wedge \bar{\xi} \wedge \bar{\eta})=\sum_{\substack{\alpha, \beta \in \Delta^{-1,1} \\ \alpha<t_{i} \beta}}\left(N_{\alpha,-\beta}^{2}+<\alpha, \beta>+N_{\alpha, \beta}^{2}\right) \cdot\left|\xi^{\alpha} \eta^{\beta}-\xi^{\beta} \wedge \eta^{\alpha}\right|^{2}
$$

Proof. As usual :

$$
\pi^{*} p_{1}(\mathcal{F})=-c_{2}\left(\mathcal{F}_{\mathfrak{k}_{\mathbb{C}}}\right)=-\frac{1}{8 \pi^{2}} \operatorname{Tr} \Theta_{D}\left(\mathfrak{k}_{\mathbb{C}}\right)^{2}
$$

For simplicity we will denote by $\Xi$ the $(2,2)$-form $-8 \pi^{2} \cdot \pi^{*} p_{1}(\mathcal{F})$. As recalled in corollary 3 for any $\xi \in \mathfrak{g}_{\mathbb{C}}^{-1,1}$ one has

$$
\Theta_{D}\left(\mathfrak{k}_{\mathbb{C}}\right)(\xi \wedge \bar{\xi})=-\operatorname{ad}_{\mathfrak{k}_{\mathbb{C}}}[\xi, \sigma(\xi)] \in \operatorname{End} \mathcal{F}_{\mathfrak{k}_{\mathbb{C}}} .
$$

Notice that $\sigma=-\tau$ on $\mathfrak{g}_{\mathbb{C}}^{-1,1}$. Thus for any $\xi, \eta \in \mathfrak{g}_{\mathbb{C}}^{-1,1}$ we get :

(9) $\left.\Xi(\xi \wedge \eta \wedge \bar{\xi} \wedge \bar{\eta})=\operatorname{Tr}\left(\operatorname{ad}_{\mathfrak{k}_{\mathbb{C}}}[\xi, \tau(\xi)] \operatorname{ad}_{\mathfrak{k}_{\mathbb{C}}}[\eta, \tau(\eta)] \mid \mathfrak{k}_{\mathbb{C}}\right)-\operatorname{Tr}\left(\operatorname{ad}_{\mathfrak{k}_{\mathbb{C}}}[\xi, \tau(\eta)] \operatorname{ad}_{\mathfrak{k}_{\mathbb{C}}}[\eta, \tau(\xi)] \mid \mathfrak{k}_{\mathbb{C}}\right)\right)$, which can be rewritten as :

$$
\Xi(\xi \wedge \eta \wedge \bar{\xi} \wedge \bar{\eta})=B_{\mathfrak{k}_{\mathbb{C}}}([\xi, \tau(\xi)],[\eta, \tau(\eta)])-B_{\mathfrak{k}_{\mathbb{C}}}([\xi, \tau(\eta)],[\eta, \tau(\xi)]) .
$$

Let $\alpha, \beta, \gamma, \varepsilon \in \Delta^{-1,1}$ and $e_{\alpha}, e_{\beta}, e_{\gamma}, e_{\varepsilon} \in \mathfrak{g}_{\mathbb{C}}^{-1,1}$ the corresponding vectors of our Weyl basis. Thus

$$
\begin{aligned}
\Xi_{\alpha \beta \overline{\gamma \varepsilon}} & =B_{\mathfrak{k}_{\mathbb{C}}}\left(\left[e_{\alpha}, e_{-\gamma}\right],\left[e_{\beta}, e_{\varepsilon}\right]\right)-B_{\mathfrak{k}_{\mathbb{C}}}\left(\left[e_{\alpha}, e_{-\varepsilon}\right],\left[e_{\beta}, e_{-\gamma}\right]\right) \\
& =N_{\alpha,-\gamma} N_{\beta,-\delta} B_{\mathfrak{k}_{\mathbb{C}}}\left(e_{\alpha-\gamma}, e_{\beta-\varepsilon}\right)-N_{\alpha,-\varepsilon} N_{\beta,-\gamma} B_{\mathfrak{k}_{\mathbb{C}}}\left(e_{\alpha-\varepsilon}, e_{\beta-\gamma}\right),
\end{aligned}
$$

with the convention $e_{\alpha-\alpha}=h_{\alpha}$.

Thus :

$$
\Xi_{\alpha \beta \overline{\gamma \varepsilon}}= \begin{cases}N_{\alpha,-\gamma} N_{\beta,-\delta}-N_{\alpha,-\varepsilon} N_{\beta,-\gamma} & \text { if } \alpha-\gamma=\beta-\varepsilon \text { and } \alpha \neq \gamma \\ N_{\alpha,-\beta}^{2}+<\alpha, \beta> & \text { if } \alpha=\gamma \text { and } \beta=\varepsilon \\ 0 & \text { otherwise. }\end{cases}
$$

This expression can be simplified using the following :

Lemma 2. 17, p.147] Let $\alpha, \beta, \gamma$ and $\varepsilon$ be 4 roots in $\Delta$, no two of which have sum 0 . Then

$$
N_{\alpha, \beta} N_{\gamma, \varepsilon}+N_{\beta, \gamma} N_{\alpha, \varepsilon}+N_{\gamma, \alpha} N_{\beta, \varepsilon}=0 \text {. }
$$

Let us apply this lemma to the first case of equation (11) and the sum of roots $\alpha+\beta-\gamma-\delta=0$. Using $N_{-\gamma, \alpha}=-N_{\alpha,-\gamma}$ and $N_{-\gamma,-\varepsilon}=-N_{\gamma, \varepsilon}$ one obtains :

\section{Lemma 3.}

$$
\Xi_{\alpha \beta \overline{\gamma \varepsilon}}= \begin{cases}-N_{\alpha, \beta} N_{\gamma, \varepsilon} & \text { if } \alpha-\gamma=\beta-\varepsilon \text { and } \alpha \neq \gamma \\ N_{\alpha,-\beta}^{2}+<\alpha, \beta> & \text { if } \alpha=\gamma \text { and } \beta=\varepsilon \\ 0 & \text { otherwise. }\end{cases}
$$


Let now $\xi, \eta \in \mathfrak{g}_{\mathbb{C}}^{-1,1}$. We write $\xi=\sum_{\alpha} \xi^{\alpha} e_{\alpha}$ and $\eta=\sum_{\beta} \eta^{\beta} e_{\beta}$, where for simplicity of notations all the indices $\alpha, \beta, \gamma, \varepsilon$ from now on vary in $\Delta^{-1,1}$. Thus

$$
\xi \wedge \eta=\sum_{\alpha<{ }_{t} \beta}\left(\xi^{\alpha} \eta^{\beta}-\xi^{\beta} \eta^{\alpha}\right) \cdot e_{\alpha} \wedge e_{\beta}
$$

We deduce from the lemma :

$$
\begin{aligned}
\Xi(\xi \wedge \eta \wedge \overline{\xi \wedge \eta})=- & \sum_{\substack{\alpha+\beta=\gamma+\varepsilon \\
\alpha<t \beta \\
\gamma<t \\
\alpha \neq \gamma}}\left(\xi^{\alpha} \eta^{\beta}-\xi^{\beta} \eta^{\alpha}\right) \cdot \overline{\left(\xi^{\gamma} \eta^{\varepsilon}-\xi^{\varepsilon} \eta^{\gamma}\right)} \cdot N_{\alpha, \beta} \cdot N_{\gamma, \varepsilon} \\
& +\sum_{\alpha<{ }_{t} \beta}\left|\xi^{\alpha} \eta^{\beta}-\xi^{\beta} \eta^{\alpha}\right|^{2} \cdot\left(N_{\alpha,-\beta}^{2}+<\alpha, \beta>\right)
\end{aligned}
$$

and thus

$$
\begin{aligned}
\Xi(\xi \wedge \eta \wedge \overline{\xi \wedge \eta})=- & \sum_{\gamma<{ }_{t} \varepsilon} \frac{\left(\xi^{\gamma} \eta^{\varepsilon}-\xi^{\varepsilon} \eta^{\gamma}\right)}{\bar{\xi}} \cdot N_{\gamma, \varepsilon} \cdot\left\{\sum_{\substack{\alpha<{ }_{t} \beta \\
\alpha \neq \gamma \\
\alpha+\beta=\gamma+\varepsilon}}\left(\xi^{\alpha} \eta^{\beta}-\xi^{\beta} \eta^{\alpha}\right) N_{\alpha, \beta}\right\} \\
& +\sum_{\alpha{ }_{t} \beta}\left|\xi^{\alpha} \eta^{\beta}-\xi^{\beta} \eta^{\alpha}\right|^{2} \cdot\left(N_{\alpha,-\beta}^{2}+<\alpha, \beta>\right)
\end{aligned}
$$

Suppose now that $[\xi, \eta]=0$. Thus $\sum_{\alpha{ }_{t} \beta}\left(\xi^{\alpha} \eta^{\beta}-\xi^{\beta} \eta^{\alpha}\right) \cdot\left[e_{\alpha}, e_{\beta}\right]=0$ which is

$$
\sum_{\substack{\alpha<{ }_{\text {tixed }} \\ \alpha+\beta \text { fixed }}}\left(\xi^{\alpha} \eta^{\beta}-\xi^{\beta} \eta^{\alpha}\right) \cdot N_{\alpha, \beta}=0
$$

which implies for any $\gamma, \varepsilon \in \Delta$ ':

$$
\sum_{\substack{\alpha<{ }^{\alpha} \\ \alpha+\beta=\gamma+\varepsilon \\ \alpha \neq \gamma}}\left(\xi^{\alpha} \eta^{\beta}-\xi^{\beta} \eta^{\alpha}\right) \cdot N_{\alpha, \beta}=-\left(\xi^{\gamma} \eta^{\varepsilon}-\xi^{\beta} \eta^{\gamma}\right) \cdot N_{\gamma, \varepsilon}
$$

Replacing

$$
\sum_{\substack{\alpha<t \beta \\ \alpha+\beta=\gamma+\varepsilon \\ \alpha \neq \gamma}}\left(\xi^{\alpha} \eta^{\beta}-\xi^{\beta} \eta^{\alpha}\right) \cdot N_{\alpha, \beta}
$$

by

$$
-\left(\xi^{\gamma} \eta^{\varepsilon}-\xi^{\beta} \eta^{\gamma}\right) \cdot N_{\gamma, \varepsilon}
$$

in equation (13) we obtain theorem 11.

6.2. Proof of theorem 4. By theorem 11 it is equivalent to showing that

$$
N_{\alpha,-\beta}^{2}+<\alpha, \beta>+N_{\alpha, \beta}^{2} \geq 0
$$

for any roots $\alpha, \beta \in \Delta^{-1,1}$. Otherwise $<\alpha, \beta><0$. But then $\alpha, \alpha+\beta, \cdots, \alpha+k \beta$ is the $\beta$-string through $\alpha$, with

On the other hand by [17, p.151] :

$$
k=-2 \frac{<\alpha, \beta>}{<\beta, \beta>} .
$$

$$
N_{\alpha, \beta}^{2}=\frac{k}{2}<\alpha, \alpha>=-<\alpha, \beta>
$$


Thus

$$
N_{\alpha,-\beta}^{2}+<\alpha, \beta>+N_{\alpha, \beta}^{2}=N_{\alpha,-\beta}^{2} \geq 0
$$

\section{Proof of Corollary 1 and theorem 1}

We first prove corollary 1, Let $\gamma \in \Phi^{c}$ be the unique root whose associated block $\mathcal{F}_{\mathfrak{g}_{\mathbb{C}, \gamma}^{-1,1}}:=$ $\bigoplus_{\alpha \in(\gamma+<\theta>) \cap \Delta_{+}} \mathfrak{g}_{\alpha}$ is contained in $\operatorname{Hom}\left(E^{k}, E^{k-1}\right)$. Let $\Psi^{\gamma} \subset \Psi$ be the connected component of the vertex $\gamma$ in the subdiagram of the Dynkin diagram of $\Psi$ generated by $\Phi \cup\{\gamma\}$. Let $\Delta^{\gamma}$ be the root system associated to $\Psi^{\gamma}$ and $\Delta_{+}^{\gamma} \subset \Delta_{+}$the set of positive roots in $\Delta^{\gamma}$. The root system $\delta^{\gamma}$ is of Hermitian type, its unique positive non-compact root in $\Psi^{\gamma}$ is $\gamma$ and the set $(\gamma+<\theta>) \cap \Delta_{+}$ identifies with the set of positive non-compact roots of this system.

Suppose now $r_{k}=1$. This means that $\Psi^{\gamma} \backslash\{\gamma\}$ is still connected and the roots in $(\gamma+<\theta>) \cap \Delta_{+}$ are strictly ordered. Let $\alpha, \beta$ be two such roots, without loss of generality we can assume $\alpha \geq \beta$. Thus

$$
<\alpha, \beta>=<\beta, \beta>+<\alpha-\beta, \beta>\geq<\beta, \beta>>0
$$

as $\alpha-\beta$ is still a positive combination of positive roots (and $\beta$ too).

This proves that for any $\xi, \eta \in \mathfrak{g}_{\mathbb{C}, \gamma}^{-1,1}$ satisfying $[\xi, \eta]=0$ one has

$$
\pi^{*} p_{1}(\mathcal{F})(\xi \wedge \eta \wedge \bar{\xi} \wedge \bar{\eta})<0
$$

except if $\xi^{\alpha} \eta^{\beta}=\xi^{\beta} \eta^{\alpha}$ for every $\alpha, \beta \in(\gamma+<\theta>) \cap \Delta_{+}$, i.e. if $\xi$ and $\eta$ are proportional.

This immediately implies that if $\bar{f}_{\rho}^{*} p_{1}(\mathcal{F})$ vanishes identically on $X$ then $q_{k} \circ f_{\rho}: \tilde{X} \longrightarrow D_{k}$ has rank 1 , where $D_{k}$ denotes the open $G$-orbit in $\check{D}_{k}$ image of $D$ under $q_{k}$.

We now argue as in [21, section 4.5]. We apply a theorem of Kaup [12, Theorem 6] which states that there exists a normal complex curve $\Sigma$ and holomorphic maps $h: \tilde{M} \longrightarrow \Sigma, \psi: \Sigma \longrightarrow \check{D}_{k}$, with $h$ surjective, such that $f_{\rho}=\psi \circ h$ :

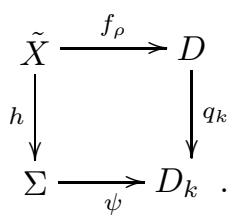

Which finishes the proof of corollary 1 .

To complete the proof of the main statement $b^{2}(\Gamma)+b^{4}(\Gamma)>0$ of theorem 1 one can assume we have a factorization as in diagram (15) (otherwise $b^{4}(\Gamma)>0$ ). We continue to argue as in [21, section 4.5]. As the map $q_{k} \circ f_{\rho}$ is superhorizontal and $D_{k}$ admits a function $\phi$ whose Levi form $L(\phi)$ is positive on the superhorizontal tangent space to $D_{k}$ (c.f. [21, prop.4.2]) the curve $\Sigma$ is not $\mathbf{P}^{1} \mathbb{C}$, in particular $\Sigma$ is aspherical. By [21, prop.4.2] the restriction to $T_{s h} D_{k}$ of the $G$-equivariant curvature form of the canonical line bundle $K_{D_{k}}$ is positive thus its pull-back under $q_{k} \circ f_{\rho}$ is a positive $(1,1)$-form on $X$ defining a non-trivial element in $H^{2}(X, \mathbb{R})$. As $q_{k} \circ f_{\rho}$ factorizes through $\Sigma$ which is aspherical, this element kills $\pi_{2}(X)$ thus belongs to the subspace $H^{2}(\Gamma, \mathbb{R}) \hookrightarrow H^{2}(X, \mathbb{R})$. Which finishes the proof of the main statement of theorem 1 
For the statement concerning $H_{b}^{4}(\Gamma, \mathbb{R})$ it is enough to notice that by [15] the continuous cohomology of $G$ lifts to bounded cohomology. The commutative diagram

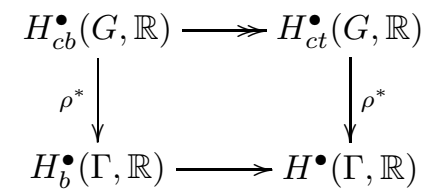

implies the result.

\section{Proof of theorem 2 AND theorem 3}

8.1. Proof of theorem 2, Suppose $\Gamma$ as in theorem 2, Let $\rho: \Gamma \hookrightarrow G L(n, \mathbb{C})$ be an unbounded embedding of $\Gamma$. As $b^{2}(\Gamma)=0$ and $\Gamma$ is an infinite Kähler group the group $\Gamma$ is schematically rigid [20, cor.3]. Arguing as in [20] we can assume that $X$ is the fundamental group of a smooth projective complex variety $X$.

By proposition $1 \rho$ is the monodromy of a $\mathbb{C V H S}$

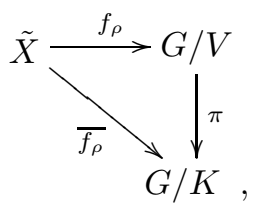

where $\mathbf{G}$ is a real form of Hodge type of the Zariski-closure of $\Gamma$ in $\mathbf{G L}(n, \mathbb{C})$. The group $G$ is non compact as $\Gamma$ is unbounded in $G L(n, \mathbb{C})$. By replacing if necessary this $\mathbb{C V H S}$ by a convenient exterior power as in corollary 1 and noticing that the corresponding monodromy still defines a linear unbounded embedding of $\rho$ we obtain from corollary 1 and its proof that the map

$$
c^{*}: H^{4}(\Gamma, \mathbb{R}) \longrightarrow H^{4}(X, \mathbb{R})
$$

deduced from the classyfing map $c: X \longrightarrow B \Gamma$ is non-zero : it maps the non-zero class $\rho^{*} p_{1}(\mathcal{F}) \in$ $H^{4}(\Gamma, \mathbb{R})$ to the non-zero De Rham class $\left[{\overline{f_{\rho}}}^{*} p_{1}(\mathcal{F})\right] \in H^{2,2}(X, \mathbb{R})$.

Using Lefschetz's hyperplane theorem we can without loss of generality assume that $X$ is a (connected) smooth projective complex surface. In this case equation (16) is equivalent to saying that

$$
c_{*}: H_{4}(M, \mathbb{R}) \simeq \mathbb{R} \longrightarrow H_{4}(\Gamma, \mathbb{R})
$$

is injective.

We proceed by contradiction to prove theorem 2. Suppose $b^{4}(\Gamma) \leq 1$. By [20, theor.1] we can assume that $b^{4}(\Gamma)=1$. By the previous remarks we obtain that

$$
c_{*}: H_{4}(M, \mathbb{R}) \simeq \mathbb{R} \stackrel{\sim}{\longrightarrow} H_{4}(\Gamma, \mathbb{R}) \simeq \mathbb{R}
$$

is an isomorphism.

We now follow closely the proof of [20, theor.1]. As in [20, section 5.1.1] we get that the universal cover $\tilde{X}$ has the homotopy type of a 2-dimensional CW-complex. But then the homological exact sequence $([20$, proposition $2,(\mathrm{a})]$

$$
H_{4}(M)_{\mathbb{Q}} \longrightarrow H_{4}(\Gamma)_{\mathbb{Q}} \longrightarrow H_{1}\left(\Gamma, \pi_{2}(M)_{\mathbb{Q}}\right) \longrightarrow H_{3}(M)_{\mathbb{Q}}
$$

still leads to

$$
H_{1}\left(\Gamma, \pi_{2}(M)_{\mathbb{Q}}\right)=0 .
$$


Following the end of the proof of [20, theor.5] we still get a contradiction to the duality property for $\Gamma$.

8.2. Proof of theorem 3. Let $G$ be a simple real Lie group of non-compact type. Matsushima proved in [24] that there exists a constant $m(G)$ depending only of the Lie algebra $\mathfrak{g}$ such that the restriction map

$$
\forall i \leq m(G), \quad H_{c t}^{i}(G, \mathbb{R}) \longrightarrow H^{i}(\Gamma, \mathbb{R})
$$

is an isomorphism for any cocompact lattice $\Gamma$ of $G$. One easily checks $\left[24\right.$ that $m(G) \geq \frac{\mathrm{rk}_{\mathbb{R}} G}{4}-1$.

For $G$ of Hodge type but not of Hermitian type we have

$$
\operatorname{dim}_{\mathbb{R}} H^{2}(\mathfrak{g}, K, \mathbb{R})=0 \quad \text { and } \quad \operatorname{dim}_{\mathbb{R}} H^{4}(\mathfrak{g}, K, \mathbb{R})=1 .
$$

Thus for $\operatorname{rk}_{\mathbb{R}} G \geq 12$ one has $b^{2}(\Gamma)=0$ and for $\operatorname{rk}_{\mathbb{R}} G \geq 20$ one has $b^{4}(\Gamma)=1$.

Moreover $\Gamma$ is an $r$-dimensional duality group, with $r=\operatorname{dim}_{\mathbb{R}}(G / K) \geq \operatorname{rk}_{\mathbb{R}} G$.

Thus theorem 2 implies theorem 3.

\section{REFERENCES}

[1] Amorós J., Burger M., Corlette K., Kotschick D., Toledo D., Fundamental groups of compact Kähler manifolds, Mathematical Surveys and Monographs 44. American Mathematical Society, Providence, RI, 1996

[2] Baily W. L., Borel A., Compactification of arithmetic quotients of bounded symmetric domains, Ann. of Math. (2) 841966 442-528

[3] Borel A., Sur la cohomologie des espaces fibrés principaux et des espaces homogènes des groupes de Lie compacts, Ann. Math. 57 (1953), 115-207.

[4] Borel A., Hirzebruch F., Characteristic classes and homogeneous spaces, Americ. Journ. of Math. 80, 2, 458-538

[5] Borel A., Wallach N., Continuous cohomology, discrete subgroups and representations of reductive groups, Second edition, Mathematical surveys and monographs 67 (2000)

[6] Bourbaki N., Groupes et algèbres de Lie, chap.4,5,6, Eléments de Mathématiques, Masson, Paris, 1981

[7] Carlson J., Toledo D., Harmonic mappings of Kähler manifolds to locally symmetric spaces, Publ. Math. IHES No. 69 (1989), 173-201

[8] Corlette K., Flat G-bundles with canonical metrics, J. Differential Geom. 28 (1988), no. 3, 361-382.

[9] Deligne P., Travaux de Griffiths. Séminaire Bourbaki 376 (juin 1970); Lecture Notes in Math 180 (SpringerVerlag 1971) pp. 213-237.

[10] Dupont J.L., Curvature and characteristic classes, Lecture Notes in Mathematics 640, Springer-Verlag (1978)

[11] Fomenko A.T., Variational principles of topology. Multidimensional minimal surface theory. Mathematics and its Applications (Soviet Series) $\mathbf{4 2}$ Kluwer Academic Publishers Group, Dordrecht, 1990

[12] Grauert H., Set theoretic complex equivalence relations, Math. Ann. 265, 1983, 137-148

[13] Griffiths P., Periods of integrals on algebraic manifolds I, Amer. Journ. of Math. 90 (1968) 568-626

[14] Griffiths P., Schmid W., Locally homogeneous complex manifolds, Acta Math. 123 (1969) 253-302

[15] Gromov M., Volume and bounded cohomology, Inst. Hautes Etudes Sci. Publ. Math. 56 (1982) 5-99

[16] Guichardet A., Wigner D., Sur la cohomologie réelle des groupes de Lie simples réels, Ann. Sci. Ecole Norm. Sup. (4) 11 (1978), no. 2, 277-292.

[17] Helgason S., Differential geometry and symmetric spaces, Academic Press, 1962.

[18] Hitchin N., The self-duality equations on a Riemann surface. Proc. London Math. Soc. (3) 55 (1987), no. 1, 59-126.

[19] Klingler B., Volume des représentations sur un corps local, GAFA 13 (2003), 1120-1160

[20] Klingler B., Kähler groups and duality, preprint available at http://people.math.jussieu.fr/ klingler/

[21] Klingler B., Koziarz J., Maubon J., On the second cohomology of Kähler groups, preprint available at http://people.math.jussieu.fr/ klingler/, submitted

[22] Kollar J., Shafarevich maps and automorphic forms. M. B. Porter Lectures. Princeton University Press, Princeton, NJ, 1995.

[23] Margulis G.A, Discrete subgroups of semisimple Lie groups, Ergebnisse der Mathematik und ihrer Grengebiete 17, Springer-Verlag, (1991)

[24] Matsushima Y., On Betti numbers of compact, locally sysmmetric Riemannian manifolds, Osaka Math. J. 14 (1962) 1-20 
[25] Salamon S., Quaternionic Kähler manifolds, Invent. Math. 67 (1982), no. 1, 143-171

[26] Simpson C., Nonabelian Hodge theory. Proceedings of the International Congress of Mathematicians, Vol. I, II (Kyoto, 1990), 747-756, Math. Soc. Japan, Tokyo, 1991

[27] Simpson C., Higgs bundles and local systems, Publ. Math. IHES 75 (1992) 5-95

[28] Simpson C., Moduli of representations of the fundamental group of a smooth projective variety I, Publ. Math. IHES 79 (1994) 47-129 ; II. Publ. Math. IHES 80 (1994), 5-79 (1995).

[29] Simpson C., The construction problem in Kähler geometry. Different faces of geometry, 365-402, Int. Math. Ser. (N. Y.), Kluwer/Plenum, New York, 2004

[30] Siu Y.T., The complex-analyticity of harmonic maps and the strong rigidity of compact Kähler manifolds, Ann. of Math. (2) 112 (1980), no. 1, 73-111.

[31] Toledo D., Projective varieties with non-residually finite fundamental group, Publ. Math. IHES 77 (1993), 103-119.

[32] Toledo D., Rigidity theorems in Kähler geometry and fundamental groups of varieties, Several complex variables (Berkeley, CA, 1995-1996), 509-533, Math. Sci. Res. Inst. Publ., 37, Cambridge Univ. Press, Cambridge, 1999

Bruno Klingler

Institut de Mathématiques de Jussieu, Paris 75013, France

e-mail : klingler@math.jussieu.fr 